\title{
Hypoglycemia Prevention and User Acceptance of an Insulin Pump System with Predictive Low Glucose Management
}

\author{
Pratik Choudhary, MD, Birthe S. Olsen, MD, Ignacio Conget, MD, \\ John B. Welsh, MD, PhD, Linda Vorrink, $\mathrm{MSc}^{5}$ and John J. Shin, $\mathrm{PhD}^{4}$
}

\begin{abstract}
Background: The MiniMed 640G sensor-augmented insulin pump system (Medtronic, Inc., Northridge, CA) can automatically suspend insulin delivery in advance of predicted hypoglycemia and restart it upon recovery. The aims of this analysis were to determine the rate at which predicted hypoglycemia was avoided with this strategy, as well as to assess user acceptance of the system and its insulin management features.

Subjects and Methods: Forty subjects with type 1 diabetes used the system for 4 weeks. We retrospectively evaluated performance of the system, using downloaded pump and sensor data, and evaluated user acceptance via questionnaires.

Results: There were 2,322 suspend before low events (2.1 per subject-day). The mean ( $\pm \mathrm{SD})$ duration of pump suspension events was $56.4 \pm 9.6 \mathrm{~min}$, and the mean subsequent sensor glucose (SG) nadir was $71.8 \pm 5.2 \mathrm{mg} / \mathrm{dL}$. SG values following $1,930(83.1 \%)$ of the predictive suspensions did not reach the preset low limit. Nadir SG values of $\leq 50$ and $\leq 60 \mathrm{mg} / \mathrm{dL}$ were seen in $207(8.9 \%)$ and $356(15.3 \%)$ of the predictive suspensions, respectively. Blood glucose (BG) and SG values before and during the study were comparable $(P>0.05)$. The mean absolute relative difference between paired SG and BG values was $10.9 \pm 13.8 \%$. Subjects felt confident using the system, agreed that it helped protect them from hypoglycemia, and wished to continue using it.

Conclusions: Automatic insulin pump suspension as implemented in the MiniMed 640G system can help patients avoid hypoglycemia, without significantly increasing hyperglycemia.
\end{abstract}

\section{Introduction} N THE CONTEXT OF SENSOR-AUGMENTED pump therapy, automatically suspending basal insulin delivery in response to low sensor glucose (SG) levels is an established way to reduce the severity and duration of hypoglycemic events. ${ }^{1-3}$ Suspending insulin delivery in response to predicted hypoglycemia has been tested in studies using various configurations of pumps, sensors, induction protocols, and algorithms. ${ }^{4-6}$

The MiniMed 640G system (Medtronic, Inc., Northridge, CA) includes a feature called SmartGuard ${ }^{\mathrm{TM}}$, which includes a predictive low glucose management algorithm, referred to as suspend before low, that suspends insulin delivery when the SG value is predicted to reach or fall below a preset low glucose limit within $30 \mathrm{~min}$ and automatically restarts basal insulin on recovery from hypoglycemia. As of September 2015, the system was commercially available in the European Union, Australia, Hong Kong, India, South Africa, Malaysia, and Chile. Settings for suspend before low and programmable alerts are customizable within multiple time blocks. Suspend before low and automatic resumption of insulin delivery can be programmed to occur with or without alerting the user. If

\footnotetext{
${ }^{1}$ King's College London, London, United Kingdom.

${ }^{2}$ Herlev Hospital, Herlev, Denmark.

${ }^{3}$ Diabetes Unit, Clinic and University Hospital, Barcelona, Spain.

${ }_{5}^{4}$ Medtronic International Trading Sàrl, Tolochenaz, Switzerland.

${ }^{5}$ Medtronic, Inc., Northridge, California.

This study is registered at ClinicalTrials.gov with clinical trial registration number NCT01991548.
}

(c) Pratik Choudhary, et al. 2016; Published by Mary Ann Liebert, Inc. This Open Access article is distributed under the terms of the Creative Commons Attribution Noncommercial License (http://creativecommons.org/licenses/by-nc/4.0/) which permits any noncommercial use, distribution, and reproduction in any medium, provided the original author(s) and the source are credited. 
the user-set low limit is reached, an alert that escalates will be triggered. We conducted a user evaluation study to estimate the system's ability to prevent predicted hypoglycemia and its acceptability to users and report our analysis here.

\section{Research Design and Methods}

Forty participants with type 1 diabetes (24 adults and 16 children) and prior experience with sensor-augmented pump therapy were enrolled across three European sites after local ethics committees' approval. Following informed consent and screening, patients were trained in use of the system, and individualized targets were set up for the "low limit" between 50 and $80 \mathrm{mg} / \mathrm{dL}(2.8-4.5 \mathrm{mmol} / \mathrm{L})$. Patients were trained by investigators and staff members at each study site in a group setting attended by Medtronic educators with the session lasting $2-3 \mathrm{~h}$. This included training on new pump features and sensor use based on the "Getting Started Guide for MiniMed 640G" document (available in English at https:// www.medtronic-diabetes.com.au/customer-support/guidesand-manuals), as well as individualized settings for alerts and threshold for the SmartGuard system based on discussion between the clinicians and participants.

In addition to the pump, insulin reservoirs, and infusion sets, subjects were provided with Bayer (Indianapolis, IN) Contour ${ }^{\circledR}$ Next Link 2.4 blood glucose (BG) meters and test strips, New Generation Enlite ${ }^{\mathrm{TM}}$ sensors (Medtronic), ${ }^{7}$ and Guardian $^{\mathrm{TM}} 2$ Link transmitters (Medtronic). They used the system for 4 weeks, during which they had four phone contacts. Pump and sensor data were collected at baseline and at the end of study, and treatment satisfaction questionnaires and free text comments were collected at the end of the study.

The SmartGuard system suspends insulin delivery if the SG level is predicted to drop below $20 \mathrm{mg} / \mathrm{dL}$ (1.1 mmol/L) above the preset low limit within the next $30 \mathrm{~min}$. Basal insulin is automatically resumed once the $\mathrm{SG}$ value was at least $20 \mathrm{mg} / \mathrm{dL}(1.1 \mathrm{mmol} / \mathrm{L})$ above the preset low limit and predicted to be at least $40 \mathrm{mg} / \mathrm{dL}(2.2 \mathrm{mmol} / \mathrm{L})$ above it within $30 \mathrm{~min}$ and insulin delivery has been suspended for at least $30 \mathrm{~min}$. The user can manually restart basal delivery at any time, and basal insulin delivery is automatically resumed after a maximum of $120 \mathrm{~min}$ regardless of the SG level. Suspend before low events were categorized according to their duration ( $<30 \mathrm{~min}, 30$ to $<90 \mathrm{~min}$, or $\geq 90 \mathrm{~min}$ ) and starting time (daytime [08:00-22:00 h] or nighttime [22:0008:00 h]). Paired SG and BG values were used for accuracy statistics. BG values used for calibrating the sensor were excluded from accuracy calculations.

\section{Results}

Thirty-nine subjects completed the study; one subject withdrew because of a device malfunction - an internal battery failure that caused an alarm but did not lead to an adverse event.

Subjects' mean $( \pm$ SD) age was $31.7 \pm 17.1$ years. Diabetes duration was $17.2 \pm 13.3$ years. Glycosylated hemoglobin level was $7.6 \pm 0.9 \%(59.6 \pm 9.5 \mathrm{mmol} / \mathrm{mol})$. Body mass index was $23.5 \pm 4.0 \mathrm{~kg} / / \mathrm{m}^{2}$. Fifty-five percent were male.

The predictive low glucose management feature was used for an average of 29.4 \pm 5.0 days. There were 2,322 suspend before low events, a rate of 2.1 per subject per $24 \mathrm{~h}$. The most common time for any pump suspension event was in the early afternoon, whereas the most common time for 2-h pump sus- pension events was at night. The overall mean $( \pm$ SD) duration of a suspend before low event was 56.4 \pm 9.6 min (median, 57.9 [interquartile range (IQR), 48.8-63.6] $\mathrm{min}$ ). The mean duration of daytime events was significantly shorter than that of nighttime events $(50.2 \pm 10.6$ vs. $69.0 \pm 10.8 \mathrm{~min} ; P<0.0001)$. The mean subsequent $\mathrm{SG}$ nadir was $71.8 \pm 5.2 \mathrm{mg} / \mathrm{dL}(4.0 \pm 0.3 \mathrm{mmol} / \mathrm{L})$; the median was 71.8 (IQR, 67.7-76.2) $\mathrm{mg} / \mathrm{dL}(4.0[3.8-4.2]$ $\mathrm{mmol} / \mathrm{L})$. Nadir SG values of $\leq 60 \mathrm{mg} / \mathrm{dL}(\leq 3.3 \mathrm{mmol} / \mathrm{L})$ were observed in $356(15.3 \%)$ of the predictive suspensions, and of these, the mean duration that $S G$ values remained $\leq 60 \mathrm{mg} / \mathrm{dL}$ was $36.1 \pm 23.6 \mathrm{~min}$, and the median (IQR) duration was 30 $(20-45) \mathrm{min}$. Nadir SG values of $\leq 50 \mathrm{mg} / \mathrm{dL}(\leq 2.8 \mathrm{mmol} / \mathrm{L})$ were observed in 207 (8.9\%) of the predictive suspensions, and of these, the mean duration that $\mathrm{SG}$ values remained $\leq 50 \mathrm{mg} / \mathrm{dL}$ was $25.3 \pm 20.8 \mathrm{~min}$, and the median (IQR) duration was $20(10-35) \mathrm{min}$.

Figure 1A shows the trajectory of SG values surrounding pump suspension events lasting $<30,30$ to $<90$, and $\geq 90 \mathrm{~min}$, normalized so that pump suspensions started at time 0 . The line at $54 \mathrm{mg} / \mathrm{dL}(3.0 \mathrm{mmol} / \mathrm{L})$ shows the most commonly used low limit value. In all of the 329 events lasting $<30 \mathrm{~min}$, insulin delivery was resumed manually. Of the 1,555 events lasting 30 to $<90 \mathrm{~min}$, insulin delivery was resumed manually in 291 events (19\%) and automatically in 1,264 events (81\%). Of the 438 events lasting $\geq 90 \mathrm{~min}$, insulin delivery was resumed manually in 50 events $(11 \%)$ and automatically in 388 events (89\%).

Figure 1B shows the number of suspend before low events according to starting time, as well as the proportion of those that were followed by one or more SG values at or below the preset threshold value. After $83.1 \%$ of the suspend before low events, the SG value did not reach the preset low limit; this rate was similar for events starting in the daytime and nighttime hours.

Only 18 subjects had prestudy SG values available for comparison with values collected during the study; 38 subjects had prestudy BG values available for comparison. Mean glucose values before and during the study were not significantly different, whether obtained from the sensors (SG values before and during, $158.9 \pm 17.1$ and $162.1 \pm 20.9 \mathrm{mg} /$ $\mathrm{dL}[8.8 \pm 1.0$ and $9.0 \pm 1.2 \mathrm{mmol} / \mathrm{L}]$, respectively; $P>0.05)$ or the meters (BG values before and during, 176.9 \pm 32.5 and $175.2 \pm 25.9 \mathrm{mg} / \mathrm{dL}[9.8 \pm 1.8$ and $9.7 \pm 1.4 \mathrm{mmol} / \mathrm{L}]$, respectively; $P>0.05)$. Of the $2,662 \mathrm{SG} / \mathrm{BG}$ paired points collected during the study, the mean $( \pm \mathrm{SD})$ absolute relative difference (ARD) between them was $10.9 \pm 13.8 \%$, and the median ARD was $6.2 \%$. Of the 274 paired points for which the BG value was $\geq 60$ and $\leq 90 \mathrm{mg} / \mathrm{dL}$, the mean $( \pm \mathrm{SD}$ ) and median ARD values were $15.1 \pm 13.5 \%$ and $12.2 \%$, respectively.

Questionnaires estimated the extent of agreement or disagreement with various statements using a 7-point scale ranging from 1 (strong disagreement) to 7 (strong agreement). All subjects felt confident using the suspend before low feature. Mean and median responses were between 6 and 7 for "The suspend by sensor features in this pump system provide more protection from lows," "I am able to better control my glucose levels with this system," and "The suspend before low feature helps protect against lows overnight." Each of these questions asked users to compare the MiniMed 640G system with their previous pump system without those features. Additional end-of-study results related to subjects' use of and preference for the system and its individual features are 

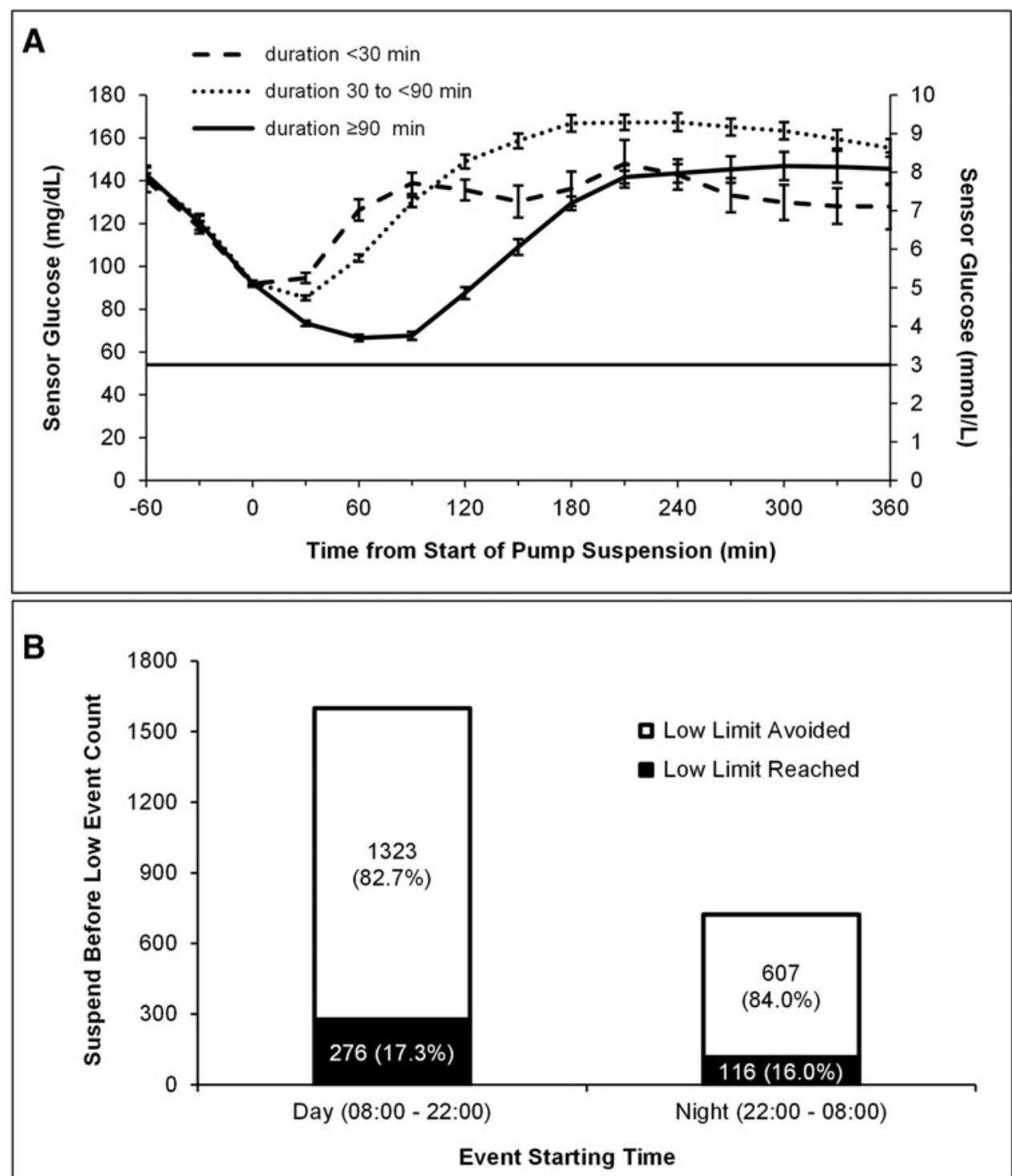

FIG. 1. (A) Mean and SD of sensor glucose trajectories throughout suspend before low events lasting $<30,30$ to $<90$, and $\geq 90 \mathrm{~min}$. The line at $54 \mathrm{mg} / \mathrm{dL}(3.0 \mathrm{mmol} / \mathrm{L})$ represents the most commonly used threshold value. (B) Number and proportion of nadir sensor glucose values following suspend before low events that were greater than the preset low limit (white) or were at or below the preset low limit (black).

available in Supplementary Table S1 (Supplementary Data are available online at www.liebertonline.com/dia). There was one device malfunction but no device-related adverse events. All four adverse events (one urinary tract infection, one case of eczema under the sensor adhesive, one case of allergic reaction to sensor overtape, and one common cold) were mild.

\section{Discussion}

These data suggest that the suspend before low feature of SmartGuard, as implemented in the MiniMed 640G pump system, is well tolerated and can prevent many hypoglycemic events in patients with type 1 diabetes. Other system components, including the New Generation Enlite sensors, performed well. The high rate at which sensor-detected hypoglycemic events were avoided is consistent with a previous study of the algorithm in which hypoglycemia induced by insulin bolus administration or exercise. ${ }^{8}$ A previous randomized controlled trial of a similar system used at night ${ }^{9}$ showed that nocturnal suspensions substantially reduced overnight hypoglycemia without conferring an increased risk of morning ketosis, suggesting that routine measurement of ketones is not necessary in the context of automated pump suspension. ${ }^{10}$ A recent study of the $640 \mathrm{G}$ algorithm implemented on a different hardware configuration $^{1}$ studied 10 patients in whom increased basal insulin delivery was intended to induce hypoglycemia; the authors concluded that use of the algorithm had the potential to reduce overnight hypoglycemia in patients on insulin pump therapy. In contrast to these earlier studies, this is the first to evaluate the commercially available system.

Routine use of the system was not associated with glycemic deterioration as measured by prestudy and study phase $\mathrm{BG}$ and $\mathrm{SG}$ values. The minimum automated suspend duration is $30 \mathrm{~min}$, so events terminated within $30 \mathrm{~min}$, as shown 
by the dashed line in Figure 1A, were terminated early by the user. These are followed by a faster and higher-amplitude rise in glucose level, likely due to carbohydrate ingestion. In contrast, the longest suspends (those lasting between 90 and $120 \mathrm{~min}$ ), shown with the continuous line, were frequently terminated by the pump algorithm and were associated with a more gradual recovery and less late hyperglycemia, possibly due to automated resumption of insulin.

This was a short observational study, and the lack of a control group prevents comparison of hypoglycemia prevention rates that might have been obtained without predictive pump suspension. The lack of a requirement for BG confirmation of SG values surrounding pump suspension events is a study limitation that precludes an estimate of the system's false-positive rate. The false-positive rate and the duration of pump suspensions depend in part on sensor accuracy in the low range, emphasizing the importance of continued improvements in sensor design. However, the rate of hypoglycemia, and in particular nocturnal hypoglycemia, was low with this system, and there was a high degree of user satisfaction recorded with the questionnaires. In particular, the feeling of safety at night was commented on strongly. The utility, feeling of nighttime security, and desire to continue using the system reported by subjects in this user evaluation study are similar to results from an earlier study of the Veo system and its low glucose suspend feature. ${ }^{2}$ Carbohydrate intake in response to symptomatic hypoglycemia was not recorded, so its contribution to rebound hyperglycemia cannot be determined, although in the comments section participants reported a lower requirements for carbohydrate intake in response to hypoglycemia.

In conclusion, the MiniMed 640G is the first commercially available system with the ability to predictively suspend and automatically restart basal insulin delivery based on continuous glucose monitoring data. Automatic insulin pump suspension with the predictive algorithm implemented in the MiniMed 640G system can help patients avoid hypoglycemia, without significantly increasing hyperglycemia. It is an important step toward closed-loop systems and can be recommended for best practice routine care. The system's longterm impacts must be confirmed in further randomized controlled studies and retrospective analyses.

\section{Acknowledgments}

This study was funded by Medtronic MiniMed, Inc., Northridge, CA.

\section{Author Disclosure Statement}

P.C., B.S.O., and I.C. have received speaker fees and/or travel support from and/or serve on advisory boards for Medtronic MiniMed, Inc. J.B.W., L.V., and J.J.S. are employees of Medtronic MiniMed, Inc.

P.C., B.S.O., and I.C. collected data and reviewed and critically revised the manuscript. J.J.S. completed the analyses, wrote, and reviewed the manuscript. J.B.W. and L.V. wrote, reviewed, and edited the manuscript. J.J.S. is the guarantor of this work and, as such, had full access to all the data in the study and takes responsibility for the integrity of the data and the accuracy of the data analysis.

\section{References}

1. Bergenstal RM, Klonoff DC, Garg SK, Bode BW, Meredith M, Slover RH, Ahmann AJ, Welsh JB, Lee SW, Kaufman FR; ASPIRE In-Home Study Group: Threshold-based insulin-pump interruption for reduction of hypoglycemia. $\mathrm{N}$ Engl J Med 2013;369:224-232.

2. Choudhary P, Shin J, Wang Y, Evans ML, Hammond PJ, Kerr D, Shaw JA, Pickup JC, Amiel SA: Insulin pump therapy with automated insulin suspension in response to hypoglycemia: reduction in nocturnal hypoglycemia in those at greatest risk. Diabetes Care 2011;34:2023-2025.

3. Ly TT, Nicholas JA, Retterath A, Lim EM, Davis EA, Jones TW: Effect of sensor-augmented insulin pump therapy and automated insulin suspension vs standard insulin pump therapy on hypoglycemia in patients with type 1 diabetes: a randomized clinical trial. JAMA 2013;310:1240-1247.

4. Danne T, Tsioli C, Kordonouri O, Blaesig S, Remus K, Roy A, Keenan B, Lee SW, Kaufman FR: The PILGRIM study: in silico modeling of a predictive low glucose management system and feasibility in youth with type 1 diabetes during exercise. Diabetes Technol Ther 2014;16:338-347.

5. Buckingham B, Chase HP, Dassau E, Cobry E, Clinton P, Gage V, Caswell K, Wilkinson J, Cameron F, Lee H, Bequette BW, Doyle FJ $3^{\text {rd}}$ : Prevention of nocturnal hypoglycemia using predictive alarm algorithms and insulin pump suspension. Diabetes Care 2010;33:1013-1017.

6. De Bock MI, Dart J, George CE, Abraham M, Cooper M, Paramalingam N, Keenan B, Spital G, Roy A, Davis EA, Jones TW: Performance of a predictive insulin pump suspension algorithm for prevention of overnight hypoglycaemia [abstract]. Diabetes 2014;63:A240-A241.

7. Veeze H, Rasmussen LG, Agrawal P, Zhong A, Arietta A, Kooi N, Liabat S, Shah R, Kaufman F: Real-life performance evaluation of the New Generation Enlite ${ }^{\mathrm{TM}}$ glucose sensor in patients with diabetes mellitus. Pediatr Diabetes 2014;15:100.

8. Abraham M, Davey R, Paramalingam N, Keenan B, Ambler G, Fairchild J, Cameron F, King B, Jones T, Davis E: Prevention of hypoglycaemia with predictive low glucose management system: comparison of hypoglycaemia induction with exercise and subcutaneous bolus [abstract]. Diabetes Technol Ther 2014;16:A43.

9. Maahs DM, Calhoun P, Buckingham BA, Chase HP, Hramiak I, Lum J, Cameron F, Bequette BW, Aye T, Paul T, Slover R, Wadwa RP, Wilson DM, Kollman C, Beck RW: A randomized trial of a home system to reduce nocturnal hypoglycemia in type 1 diabetes. Diabetes Care 2014;37:1885-1891.

10. Beck RW, Raghinaru D, Wadwa RP, Chase HP, Maahs DM, Buckingham BA: Frequency of morning ketosis after overnight insulin suspension using an automated nocturnal predictive low glucose suspend system. Diabetes Care 2014; $37: 1224-1229$.

Address correspondence to: Pratik Choudhary, MD King's College London 3rd Floor Weston Education Centre Denmark Hill, London SE5 9RJ, United Kingdom

E-mail: pratik.choudhary@kcl.ac.uk 